\title{
Impedance Spectroscopy Systems: Review and An All-Digital Adaptive IIR Filtering Approach
}

\author{
Nikola Ivanisevic, Saul Rodriguez and Ana Rusu \\ KTH Royal Institute of Technology, \\ School of Information and Communication Technology, \\ Stockholm, Sweden, \{nikiva,saul,arusu\}@kth.se
}

\begin{abstract}
Impedance spectroscopy is a low-cost sensing technique that is generating considerable interest in wearable and implantable biomedical applications since it can be efficiently integrated on a single microchip. In this paper, the fundamental characteristics of the most well-known system architectures are presented, and a more robust and hardware-efficient solution is proposed. An all-digital implementation based on adaptive filtering is used for identifying the impedance parameters of a sampleunder-test. The coefficients of an infinite-impulse-response (IIR) filter are tuned by an adaptive algorithm based on pseudo-linear regression and output-error formulation. A three-level pseudorandom noise generator with a concave power spectral density is employed without deteriorating the nominal performance. Proofof-concept has been verified with behavioral simulations.
\end{abstract}

\section{INTRODUCTION}

Various electrochemical and biomedical applications often require a non-destructive sensing techniques to analyze a sample-under-test (SUT) [1], [2]. Impedance spectroscopy is such a technique in which it is possible to extract information about the SUT morphology by measuring its electrical impedance over a wide range of frequencies. For instance, depending on the application, the impedance can be interpreted as substance composition [3], tissue ischemia [4] or the freshness of food products [5].

Traditionally, bulky benchtop impedance analyzers were used as measurement systems. Nowadays, the primary function of these instruments can be integrated on a single microchip [6]. As a direct result of CMOS integration, smaller formfactor, lower power dissipation, higher functionally and sensitivity is made possible. Furthermore, these advancements have enabled a wide range of portable systems for wearable and implantable biosensing applications, which require more challenging specifications [7], [8]. Thus, simpler and robust system architectures are highly desired.

This paper presents a review of well-known system architectures for impedance spectroscopy and proposes an all-digital implementation. The proposed solution employs a digital filter that matches the response of an unknown SUT for a defined input signal. In this way, the challenging analog signal conditioning is eliminated by employing low-complexity digital signal processing.

\section{System ARChitecture}

Impedance spectroscopy refers to the measurement of magnitude and phase delay of a response signal after applying a voltage or current excitation to an SUT. The most well-known system architectures are the fast Fourier transform (FFT) approach, the frequency response analyzer (FRA) and the magnitude/phase detection (MPD) systems. In the following section, their fundamental characteristics are discussed, and a solution is proposed to overcome their limitations.

\section{A. FFT Approach}

The FFT requires a broadband excitation signal and intensive digital signal processing to perform spectral analysis on the response signal as shown in Fig. 1a. The main advantage of the FFT approach is that the impedance is measured simultaneously over the entire frequency span. Ideally, the excitation signal should have a uniformly distributed power spectral density, to simplify the processing. For instance, a single rectangular pulse has a sinc response in the frequency domain, which is a function of the pulse amplitude and duration. Although the sinc spectrum can be approximated as constant at low frequencies, the single pulse waveform is not often used since the noise floor becomes indistinguishable from the excitation signal. The most important limitation is the peak value that can be applied to the SUT and electrode contacts without producing distortion [9]. Alternately, a more complicated waveform, such as pseudo-random noise (PRN) generator or a multi-sine [2], can be carefully constructed to avoid large signal peaks. An accurate multi-sine waveform can be very challenging to implement compared to a PRN generator, but with careful frequency planning, it is possible to differentiate between the excitation frequencies and the distortion produced by the SUT nonlinearity. Therefore, more reliable results can be warranted with a multi-sine waveform.

The ability to perform a seemingly instantaneous frequency sweep is also the primary challenge in the FFT approach [10]. For instance, the dynamic range of the response signal can vary significantly over several decades of frequencies when measuring capacitive impedance. This, in turn, increases the dynamic range requirements of the analog-to-digital converter (ADC). Also, a large data array can be generated depending on the targeted frequency span and the resolution of the ADC. Lastly, if sufficient band-limiting is not applied, issues with aliasing can occur. Most of these problems are mitigated by segmenting the frequency range in smaller and more manageable slices, which are combined in post-processing but with a potentially discontinuous spectrum. Consequently, the measurement speed is sacrificed and the results are no longer simultaneous. With further segmentation, the FFT approach naturally converges to measurements at a particular frequency as is in the FRA. 


\section{B. FRA Approach}

Compared to the FFT, in the FRA, a single sine-wave is applied to the SUT and relatively simple analog signal conditioning is employed to extract the real and imaginary components of the complex response signal. Measurements are performed sequentially by sweeping the frequency of a single sine-wave, which avoids most of the issues of the FFT approach, however the measurements are relatively slow. The architecture is inspired by the direct-conversion (homodyne) radio receiver as it can be seen in Fig. 1b. The response signal is first multiplied with a pair of square waves that are 90 degrees apart (I/Q demodulation), but with the same frequency as the excitation tone. In this way, two DC components are generated after low-pass filtering, which significantly relaxes the performance requirements on the ADC. The I/Q demodulation can employ time-multiplexing of the readout circuit to reduce chip area [8], but more importantly sharing of the readout circuit prevents additional mismatches to the real and imaginary components.

Despite its simplicity, the FRA approach brings many challenges at the circuit level. The main drawback of using square waves is the multiplication of odd harmonics with the distortions from the excitation signal [11]. Thus, the total harmonic distortion of the excitation and the readout circuit limits the largest impedance detection [2], [12]. Other issues are related to the synchronization errors, clock uncertainty, and mismatches in the duty ratios. The synchronization errors create phase offsets that can be removed with calibration at the cost of reduced measurement range [4]. The clock uncertainty in the square waves can be a large source of error during the I/Q zero-crossing. Fortunately, most of the unwanted noise can be removed via intensive analog filtering. Moreover, the additional harmonics that are generated due to the duty cycle variation are also filtered. Due to its simplicity, the FRA is still the most common approach for impedance spectroscopy. Although this approach is very popular, recent developments have led to MPD systems, which overcomes the challenges in the FRA.

\section{MPD Approach}

Instead of I/Q demodulation, the MPD systems employs simple peak and zero-crossing detection circuits [3], [13], [14]. The MPD system from [3], which is shown in Fig. 1c, avoids the implementation challenges of high-frequency analog signal conditioning of the FRA and complicated digital processing of the FFT. Consequently, the resolution of the MPD approach is set by the clock frequency. Also, the main analog issues are related to the comparator design (e.g. offset, kick-back, noise, delay) and the generation of the excitation signal. Moreover, calibration is needed due to the charging time variation in the voltage reference that is used for peak detection [3].

The total harmonic distortion of the excitation signal is still a bottleneck for the measurement accuracy since it has a profound impact on the signal zero-crossing. To circumvent this recurring issue, we propose an approach that employs an inherently linear excitation signal.
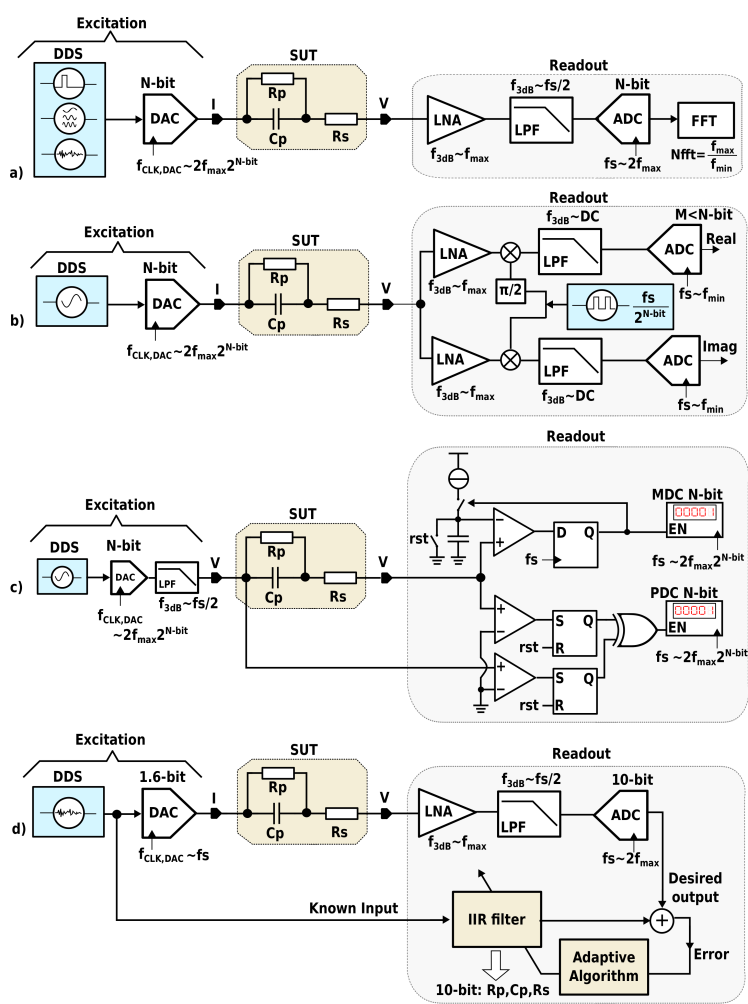

Fig. 1. System architecture of the a) FFT; b) FRA; c) MPD and d) the proposed approach.

\section{Proposed Approach - System Identification}

System identification is a digital technique that employs an adaptive filter to model the response of an unknown system over time [15]. A block diagram of the system architecture is shown in Fig. 1d. The same broadband signal (e.g. white noise) excites the SUT and the digital filter. The response of the SUT is then digitized and compared against the response of the filter to estimate the error. An adaptive algorithm then tunes the coefficients of the filter to minimize this error. In the ideal case, when the algorithm converges, the filter replicates exactly the response of an unknown SUT. However, in practice, due to implementation considerations, the error is a finite nonzero value. An infinite-impulse-response (IIR) filter is chosen since it can directly model the poles of an SUT. An alternative solution, though with a much larger number of coefficients that do not have a physical meaning, would be to use a finiteimpulse-response filter [16]. The disadvantage with using IIR filters is the potential instability, slow convergence, local and biased global minima. However, these issues can be mitigated as shown in [17]. The transfer function of the IIR filter is chosen to match that of the SUT. In this way, the impedance parameters of the SUT can be easily derived, and the approach can be validated via behavioral simulations. Although most biomaterials present different dispersion phenomena [18], the simplest circuit topology is considered for the SUT as it allows keeping the theoretical analysis at a tractable level. For a circuit implementation, the complexity of the IIR filter should be expanded to account for additional poles and zeros, e.g. due to the sample-electrode interface, the LNA, antialiasing filter, etc. 
TABLE I. SUMMARY OF PROS (+) AND CONS (-) OF DIFFERENT APPROACHES

\begin{tabular}{|lcccc|}
\hline Approach & FFT & FRA & MPD & Proposed \\
\hline Excitation bandwidth & - & + & + & - \\
System linearity & + & -- & - & ++ \\
Measurement speed & + & -- & - & + \\
System complexity & - & + & ++ & ++ \\
Robustness & -- & - & + & ++ \\
ADC/DAC resolution & -- & $+/-$ & - & $-/+$ \\
ADC/DAC clock rate & - & $+/-$ & -- & $-/+$ \\
\hline
\end{tabular}

The Laplace transform of the considered SUT, consisting of $R_{S}, R_{P}$ and $C_{P}$ parameters as shown in Fig. 1, is given by:

$$
\operatorname{SUT}(s)=\left(R_{S}+R_{P}\right) \frac{1+s \frac{R_{S} R_{P}}{R_{S}+R_{P}} C_{P}}{1+s R_{P} C_{P}},
$$

The transfer function of the IIR filter is then calculated with the $c 2 d$ MATLAB function and is given by:

$$
\operatorname{IIR}(z)=\frac{b_{2} z+b_{1}}{z-a_{1}},
$$

Based on (1) and the IIR filter coefficients, $b_{2}, b_{1}$ and $a_{1}$, the impedance parameters can be expressed as:

$$
\begin{aligned}
R_{S} & =\frac{b_{2}}{G_{m, D A C}}, \\
R_{P} & =\frac{a_{1} R_{S}+b_{1} / G_{m, D A C}}{1-a_{1}}, \\
C_{P} & =\frac{T_{S}}{R_{P}} \frac{-1}{\ln \left(a_{1}\right)},
\end{aligned}
$$

where $G_{m, D A C}$ is the transconductance of the DAC. For the purpose of continuous to discrete-time transformation, the impedance parameters were treated as constant across the frequency range, however they may vary due to different dispersion mechanisms. The proposed approach can be extended to include non-linear behavior of the SUT by applying more advanced non-linear adaptive filtering algorithms, which are commonly used in modern communications systems [15].

The adaptive algorithm is formulated based on the outputerror since it requires only the present and past values of the known input, e.g. the excitation signal. Pseudo-linear regression (PLR) is used for the adaptive algorithm implementation due to its simplicity and self-stabilizing feature [17]. The computational complexity of the PLR is comparable to that of the recursive-least-squares algorithm which is $\mathcal{O}(M+N)^{2}$, where $M$ is the number of tunable coefficients in the numerator $\left(b_{2}, b_{1}\right)$ and $N$ in the denumerator $\left(a_{1}\right)$ of $\operatorname{IIR}(z)$. On the other hand, the computational complexity of an FFT algorithm is $\mathcal{O}\left[N_{F F T} \cdot \log \left(N_{F F T}\right)\right]$, where $N_{F F T}$ is the number of points in the FFT. The $N_{F F T}$ is equal to the ratio of the highest to the lowest excitation frequency, which can be several orders of magnitude in most applications. Therefore, the complexity of the FFT is much larger than of the PLR algorithm for the considered SUT and the same ADC resolution. Furthermore, compared to the FFT based systems, adaptive filters can provide more reliable measurements for lower signal-to-noise ratios if a complex nonlinear least square method is used in data processing [19]. A summary of pros and cons for each of the analyzed approaches is shown in Table I.

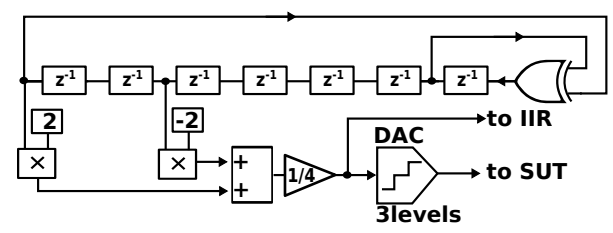

Fig. 2. Block diagram implementation of the excitation waveform.

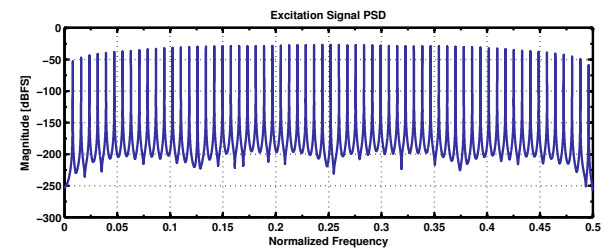

Fig. 3. Power spectral density of the excitation waveform.

\section{Simulation Results}

The proposed approach has been implemented at system level in MATLAB/Simulink. The SUT is modeled with arbitrarily chosen parameters, $R_{S}=1 \mathrm{k} \Omega, R_{P}=1 \mathrm{M} \Omega$ and $C_{P}=4 \mathrm{nF}$, that are within the range of experimentally reported impedance magnitudes [1], [8], [11], [12] For the PRN generator, a DAC with three levels was sufficient to maintain nominal performance. The noise waveform is implemented as a modified 7-bit linear feedback shift register (LFSR) as shown in Fig. 2. Compared to a conventional LFSR, the proposed implementation has a concave shape in the power spectral density as it can be seen in Fig. 3. In this way, the response signal is less likely to produce non-linearity at low frequencies where the SUT impedance is large. The transconductance of the digital-to-analog converter (DAC), $G_{m, D A C}$, is chosen approximately to be $1 / R_{S}$ to ensure that the ADC is not overloaded, assuming the DAC and ADC have the same fullscale range. In a circuit implementation, an amplifier before the ADC is recommended to allow for a smaller DAC fullscale range due to non-linearity considerations of the SUT and electrode contacts. An ADC with 10-bit resolution is used for simulations since most applications target approximately a measurement accuracy in the order of $1 \%$.

Noise and nonlinearity of the DAC and ADC were modeled as well as the thermal noise of the SUT to evaluate the tolerance of the adaptive algorithm. The root-mean-squared values of the ADC and DAC noise are proportional to $\mathrm{V}_{L S B}$ and $2 \mathrm{~V}_{L S B}$, respectively. A larger amount of noise can be tolerated in the DAC due to the low-pass transfer function of the SUT. The total harmonic distortion (THD) of the DAC should be at least $-66 \mathrm{~dB}$, which is not a demanding requirement considering that a 3 -level DAC is quasi-linear. On the other hand, the ADC should have a THD of at least $-72 \mathrm{~dB}$ to maintain the nominal performance. The sampling jitter should be smaller than $0.5 \%$ of the ADC clock period. The behavior of the system, after it has entered steady-state, is shown in Fig. 4. The adaptive filter reaches steady-state after approximately 300 clock cycles for the forgetting factor set to 0.9999 . Note, the input repeats every 127 clock cycles due to the LFSR implementation. The filter output closely follows the digitized response signal of the SUT. The output-error, between the desired and filter output, is mostly bounded to 

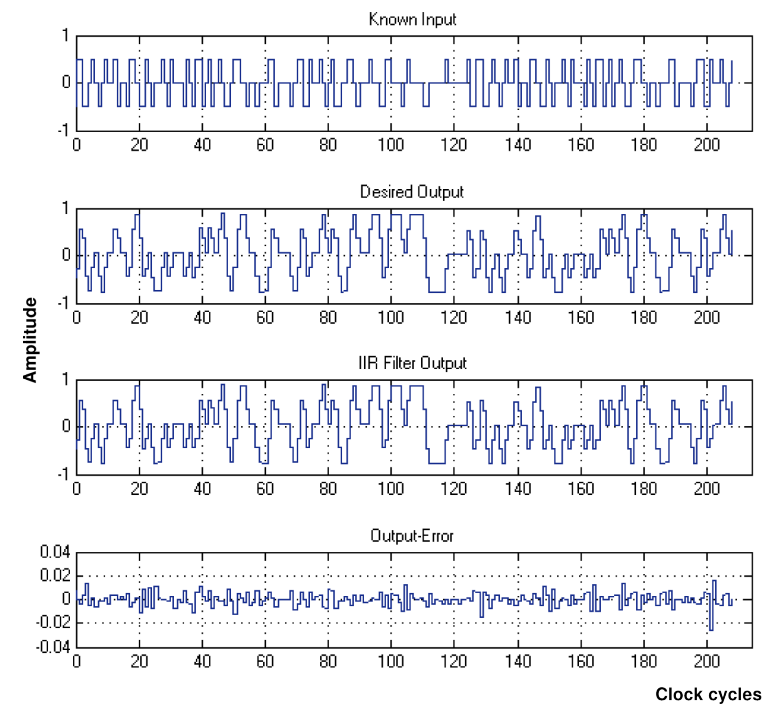

Fig. 4. Normalized waveforms of the known input, the desired and IIR filter output, and the output-error normalized to the ADC full-scale value.
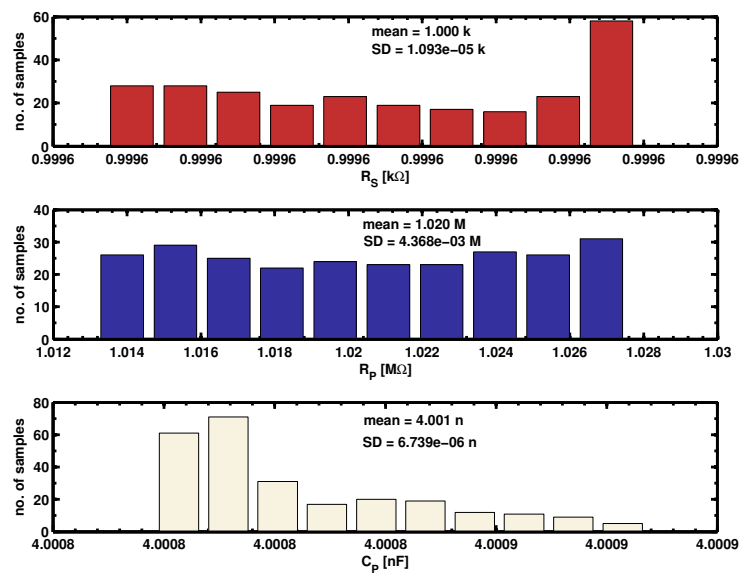

Fig. 5. Histogram of the identified impedance parameters $R_{S}, R_{P}$ and $C_{P}$.

0.02 of the ADC full-scale, which means that the system is maintaining an accurate and stable match with the SUT. The histogram of the calculated impedance parameters during the steady-state operation of the system is shown in Fig. 5. The extracted values from the adaptive filter match closely with that of the SUT. The largest error occurs in the $R_{P}$, due to the reduced power of the excitation signal at low frequencies, and is approximately $0.428 \%(1 \sigma)$ of the mean value.

\section{CONCLUSiON}

This paper presented a review of well-known impedance spectroscopy system architectures and proposed a simple alldigital architecture based on adaptive filtering. The proposed approach benefits from the instantaneous frequency sweep of the FFT, the hardware simplicity of the FRA and the robust digital circuits of the MPD approach. The main challenge is that the transfer function of the IIR filter should be expanded to account for additional poles, zeros and non-linear behavior that occur in a real measurement system, which would increase the complexity of a circuit implementation. Nevertheless, the behavioral simulation results demonstrate that the proposed architecture can accurately identify the impedance parameters of the SUT, which is a promising result for future development.

\section{ACKNOWLEDGMENT}

The authors would like to thank the Swedish Research Council (VR) for funding this work.

\section{REFERENCES}

[1] M. Vergani et al., "Multichannel bipotentiostat integrated with a microfluidic platform for electrochemical real-time monitoring of cell cultures," IEEE Trans. Biomed. Circuits and Syst., vol. 6, no. 5, pp. 498-507, Oct 2012.

[2] H. Wi et al., "Multi-frequency electrical impedance tomography system with automatic self-calibration for long-term monitoring," IEEE Trans. Biomed. Circuits Syst., vol. 8, no. 1, pp. 119-128, Feb 2014.

[3] T. A. Chen et al., "Novel 10-bit impedance-to-digital converter for electrochemical impedance spectroscopy measurements," IEEE Trans. Biomed. Circuits Syst., vol. 11, no. 2, pp. 370-379, April 2017.

[4] A. Yufera et al., "A tissue impedance measurement chip for myocardial ischemia detection," IEEE Trans. Circuits Syst. I, Reg. Papers, vol. 52, no. 12, pp. 2620-2628, Dec 2005.

[5] M. Guermazi, O. Kanoun, and N. Derbel, "Investigation of long time beef and veal meat behavior by bioimpedance spectroscopy for meat monitoring," IEEE Sensors J., vol. 14, no. 10, pp. 3624-3630, Oct 2014.

[6] D. Bianchi et al., "CMOS impedance analyzer for nanosamples investigation operating up to $150 \mathrm{mhz}$ with sub-af resolution," IEEE J. Solid-State Circuits, vol. 49, no. 12, pp. 2748-2757, Dec 2014.

[7] A. Sun, A. G. Venkatesh, and D. A. Hall, "A multi-technique reconfigurable electrochemical biosensor: Enabling personal health monitoring in mobile devices," IEEE Trans. Biomed. Circuits Syst., vol. 10, no. 5, pp. 945-954, Oct 2016.

[8] S. Rodriguez et al., "A batteryless sensor ASIC for implantable bioimpedance applications," IEEE Trans. Biomed. Circuits Syst., vol. 10, no. 3, pp. 533-544, June 2016.

[9] E. Barsoukov and J. R. Macdonald, Impedance Spectroscopy Theory, Experiment, and Applications, 2nd ed. New Jersey: John Wiley and Sons, 2005.

[10] C. Yang, D. Rairigh, and A. Mason, "Fully integrated impedance spectroscopy systems for biochemical sensor array," in 2007 IEEE Biomed. Circuits Syst. Conf., Nov 2007, pp. 21-24.

[11] H. Jafari, L. Soleymani, and R. Genov, "16-channel CMOS impedance spectroscopy DNA analyzer with dual-slope multiplying adcs," IEEE Trans. Biomed. Circuits Syst., vol. 6, no. 5, pp. 468-478, Oct 2012.

[12] A. Manickam et al., "A CMOS electrochemical impedance spectroscopy (EIS) biosensor array," IEEE Trans. Biomed. Circuits Syst., vol. 4, no. 6, pp. 379-390, Dec 2010.

[13] H. Huang and S. Palermo, "A TDC-based front-end for rapid impedance spectroscopy," in 2013 IEEE 56th Int. Midwest Symp. Circuits Syst., Aug 2013, pp. 169-172.

[14] S. J. Kweon et al., "A CMOS low-power polar demodulator for electrical bioimpedance spectroscopy using adaptive self-sampling schemes," in 2016 IEEE Biomed. Circuits Syst. Conf., Oct 2016, pp. 284-287.

[15] P. S. R. Diniz, Adaptive Filtering: Algorithms and Practical Implementation, 2nd ed. Boston: Springer, 2008.

[16] Wang, S. et al., "Battery State Estimator Based on a Finite Impulse Response Filter," J. Electrochem. Soc., vol. 160, no. 11, pp. A1962A1970, sep 2013.

[17] J. J. Shynk, "Adaptive IIR filtering," IEEE ASSP Mag., vol. 6, no. 2, pp. 4-21, April 1989.

[18] S. Grimnes and O. G. Martinsen, "Cole electrical impedance model - a critique and an alternative," IEEE Trans. Biomed. Eng., vol. 52, no. 1, pp. 132-135, Jan 2005.

[19] D. D. Stupin et al., "Adaptive filtering to enhance noise immunity of impedance and admittance spectroscopy: Comparison with fourier transformation," Phys. Rev. Applied, vol. 7, pp. 054024/1-11, May 2017. 